\title{
Gas transfer by breaking waves
}

(a) $\frac{t}{\sqrt{g k}}=1$ Wind driven waves on the sea-air interface undergo Palas Kumar Farsoiya ${ }^{1}$, Stéphane Popinet ${ }^{2}$, Luc Deike ${ }^{1,3}$

1. Department of Mechanical and Aerospace Engineering, Princeton University, Princeton, NJ 08544, USA 2. Institut Jean Le Rond d'Alembert, CNRS UMR 7190, Sorbonne Université, Paris 75005, France

3. High Meadows Environmental Institute, Princeton University, Princeton, NJ 08544, USA

(b) $\frac{t}{\sqrt{g k}}=2$

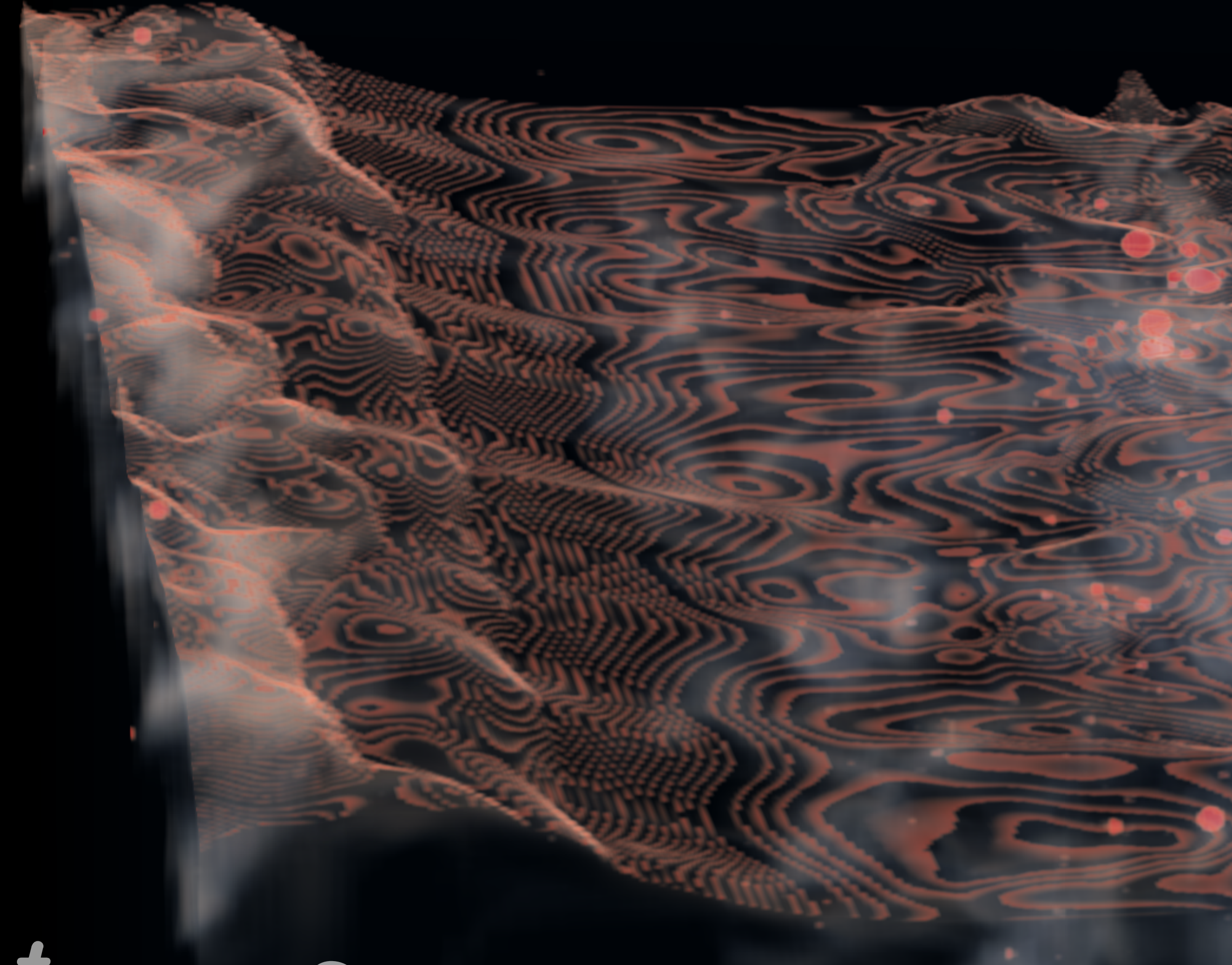

When waves are breaking at the ocean surface, air bubbles are entrained in the turbulent ocean water [Deike et al 2016, Deike \& Melville, 2018] Gas exchange is enhanced by the presence of turbulent motion around the bubbles, with thin boundary layer following the vorticity structure of the flow, as visible on the figures [and Farsoiya et. al. 2021].The total amount of gas exchange is controlled by the individual bubble transfer rate and the residence time (or rise velocity) of the bubbles in the water, both modulated by turbulence.

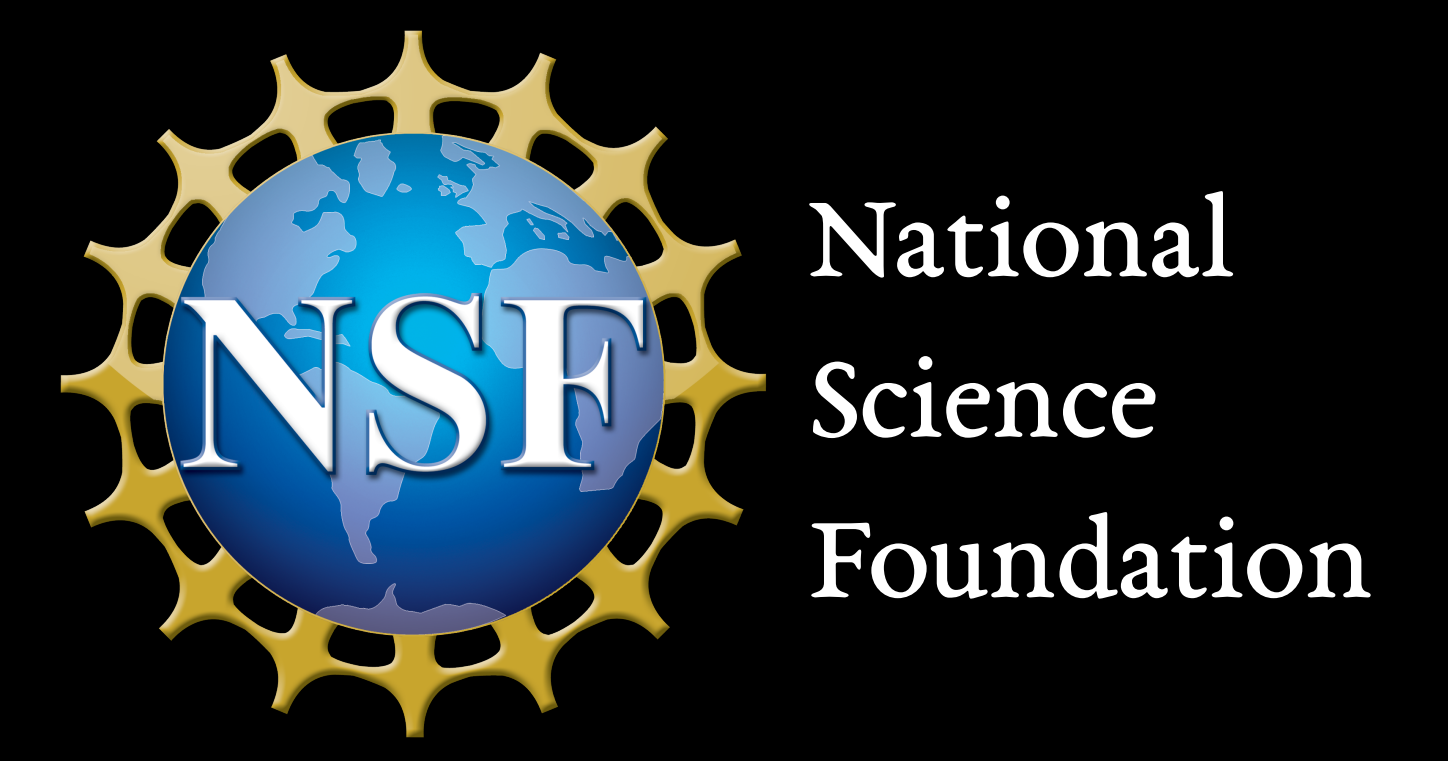

This work was supported by the NSF CAREER award 1844932, We would like to acknowledge high-performance computing support from Cheyenne (doi:10.5065/D6RX99HX) provided by NCAR's Computational and Information Systems Laboratory and by XSEDE (Stampede2) sponsored by the National Science Foundation.
The figures show concentration field (red high, white low) of a gas $(S c=100, \operatorname{Re}=40,000, B o=200)$, from Direct numerical simulations of breaking wave using Basilisk PDE solver [Popinet and Collaborators, http://basilisk.fr, Farsoiya and Popinet and Deike, 2021]

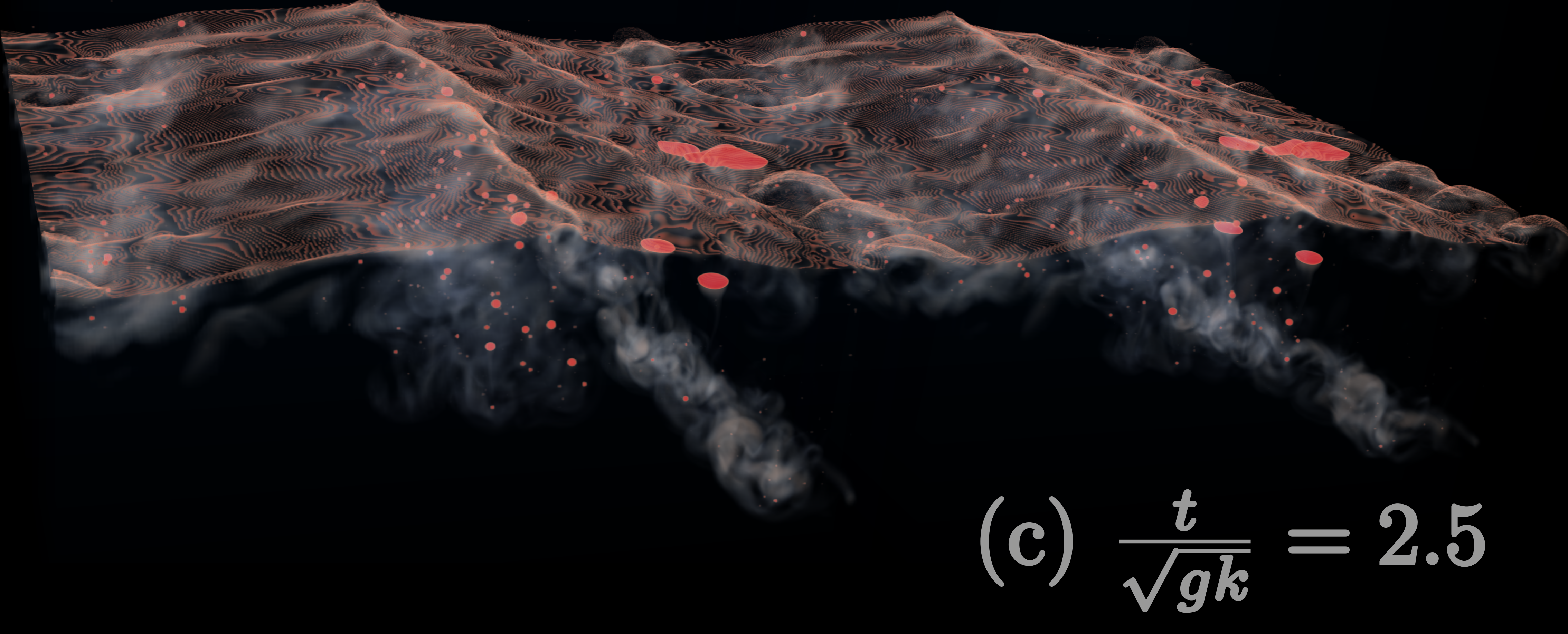

